\title{
From Experimental Data to Pole Parameters in a Model Independent Way (Fixed-t Single-Energy Partial Wave Analysis (SE PWA) and Laurent + Pietarinen Model $(L+P)$ for Pole Extraction)
}

\author{
Alfred $\breve{S}_{\text {varc }}{ }^{1,2, *}$ \\ ${ }^{1}$ Rudjer Bošković Institute, Bijenička cesta 54, P.O. Box 180, 10002 Zagreb, Croatia \\ ${ }^{2}$ TESLA BIOTECH d.o.o., Mandlova 7, 10000 Zagreb, Croatia
}

\begin{abstract}
As it has been shown in the invited talk at the NSTAR-2017 conference [1] it is in principle possible to extract pole parameters directly from experiment with minimal model dependence, and in this contribution one way to achieve it in practice by fixed-t analyticity has been demonstrated. Namely, unconstrained partialwave amplitudes obtained at discrete energies from fits even to complete sets of independent observables which are required to uniquely reconstruct reaction amplitudes do not vary smoothly with energy, and are in principle non-unique. We have demonstrated that this behavior can be ascribed to the continuum ambiguity. We have applied continuum ambiguity invariance to pseudo-scalar meson photoproduction and showed that for a complete set of pseudo-data the non-uniqueness effect can be removed through a phase rotation generating "up-to-a-phase" unique set of single energy partial wave amplitudes. Now we show that for real data this method does not work, but another approach - fixed-t analyticity solves the problem. We present the final results for the $\eta$ and $\pi^{0}$-photoproduction [2,3]. Extracting pole positions from partial wave amplitudes is the next step, and we summarize the essence of the new, Laurent + Pietarinen expansion method applicable for continuous and discrete data. It is based on applying the Laurent decomposition of partial wave amplitude, and expanding the non-resonant background into a power series of a conformal-mapping-generated, quickly converging power series obtaining the simplest analytic function with well-defined partial wave analytic properties which fits the input. Unifying both methods in succession, one constructs a model independent procedure to extract pole parameters directly from experimental data without referring to any theoretical model.
\end{abstract}

\section{Introduction}

Partial-wave analysis, a textbook method to identify resonances and attribute them quantum numbers, is a standard procedure used to analyze a wide class of experimental data (see for instance ref. [4]). In the quest for a unique set of partial-wave amplitudes, experimental programs worldwide have attempted to measure complete sets of observables needed to perform the unambiguous reactionamplitude reconstruction. Programmatic studies of photoproduction experiments at Jefferson Lab [5], Mainz [6] and Bonn [7] are now producing the data required to do complete experiments, in terms of either helicity amplitudes or multipoles, motivating a reexamination of the ambiguities associated with multipole analyses.

It is well known that single-channel physical observables remain invariant with respect to a general energyand angle-dependent phase rotation which turns out to introduce the non-uniqueness into the amplitude reconstruction. This invariance is the so-called continuum ambiguity, and has been extensively analyzed in mid-70s through the mid-80s [8-10]. The interconnection between continuum ambiguity and partial wave analysis has never been extensively discussed as most studies were made in the context of $\pi N$ elastic scattering where the optical theorem and an application of elastic unitarity practically eliminate the continuum ambiguity as a source of non-uniqueness. In the inelastic domain, up to now, the main attention has

*e-mail: svarc@irb.hr been paid only to handling angle independent phase rotations on the level of partial waves where the analytic structure remains untouched [11-13].

It is a general knowledge that as a direct consequence of continuum ambiguity the unconstrained partial-wave amplitudes obtained at discrete energies even from fits to complete sets of eight independent observables [14] which are required to uniquely reconstruct reaction amplitudes do not vary smoothly with energy, and are in principle nonunique [15]. In [1] it has been shown that for a complete set of pseudo-data this problem can be easily solved by simple phase rotations. However, in this paper we show that such an approach is not valid for the real data, and we propose another method based on imposing fixed-t analyticity which solves the problem.

Extracting poles from partial waves is yet another issue. Up to now, there was no reliable way to extract pole parameters from SE partial waves, but a new and simple single-channel method (Laurent + Pietarinen expansion) applicable for continuous and discrete partial-wave data has been recently developed [16-18]. It is based on applying the Laurent decomposition of partial wave amplitude, and expanding the non-resonant background into a power series of a conformal-mapping, quickly converging power series obtaining the simplest analytic function with welldefined partial wave analytic properties which fits the input. The method is particularly useful to analyse partial wave data obtained directly from experiment because it works with minimal theoretical bias since it avoids con- 
structing and solving elaborate theoretical models, and fitting the final parameters to the input, which is the only alternative standard procedure employed nowadays. The generalization of this method to multi- channel case is also developed and presented.

Unifying both methods in succession, one constructs a model independent procedure to extract pole parameters directly from experimental data without referring to any theoretical model.

\section{Unconstrained SE partial wave analysis (SE-PWA)}

In ref. [1] it has been in details shown that unconstrained SE-PWA for $\eta$-photoproduction is discontinuous in energy, but for the convenience of the reader let us repeat the essence.

When we perform an unconstrained SE-PWA of a complete set of pseudo-data generated from the ETA-MAID15a model [19] and put initial values to $10 \%$ randomized ETA-MAID15a model solutions, helicity amplitudes at fixed energy and fixed angle which we obtain are shown in Fig. 1.

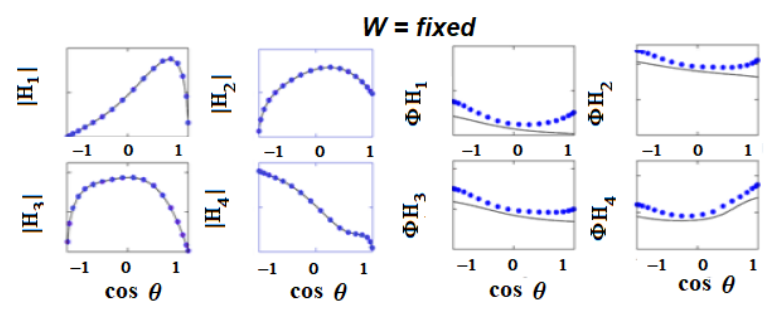

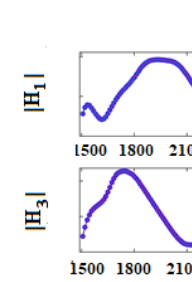

W

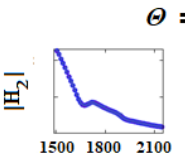

$=$ fixed

w

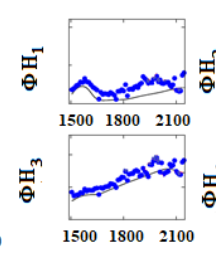

$\mathrm{w}$
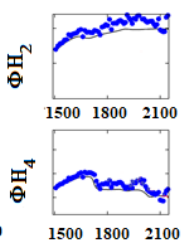

W

Figure 1: Absolute values and phases of helicity amplitudes at fixed energy and fixed angle obtained in unconstrained SE-PWA for a complete set of MAID pseudo-data [19]. Full lines are predictions of ETA-MAID15a model, and full dots are the result of unconstrained SE-PWA. The absolute scale is unimportant to see the effect.

We see that absolute values are, as expected, always reproduced, while phase is not; instead it is different and continuous at the fixed energy, but different and discontinuous at fixed angle. In both cases the reason is that no connection between phases at neighbouring energies is imposed. However, for the fixed energy, analyticity introduced through partial wave decomposition guarantees continuity, but for fixed angle no conditions whatsoever are imposed, so the result is even discontinuous.

In ref. [1] we have explicitly shown that the main reason for the problem is the unspecified and free phase at

neighbouring energies, and that rotating that phase at each energy to a continuous phase solves the problem. We introduce the following angle-dependent phase rotation simultaneously for all four helicity amplitudes:

$$
\begin{aligned}
\tilde{H}_{k}^{S E}(W, \theta) & =H_{k}^{S E}(W, \theta) \cdot e^{i \Phi_{H_{N}}^{15 a}(W, \theta)-i \Phi_{H_{N}}^{S E}(W, \theta)} \\
k & =1, \ldots, 4
\end{aligned}
$$

where $H_{N}$ is any out of four helicity amplitudes, $\Phi_{H_{N}}^{S E}(W, \theta)$ is the single-energy phase and $\Phi_{H_{N}}^{15 a}(W, \theta)$ is the continuous phase, in this case the phase of the generating solution ETA-MAID15a.

However, this method simply does not work for the real data. If we take the data base from ref. [2], and make the phase rotation with $H_{1}$ defined by Eq. (1) we obtain:
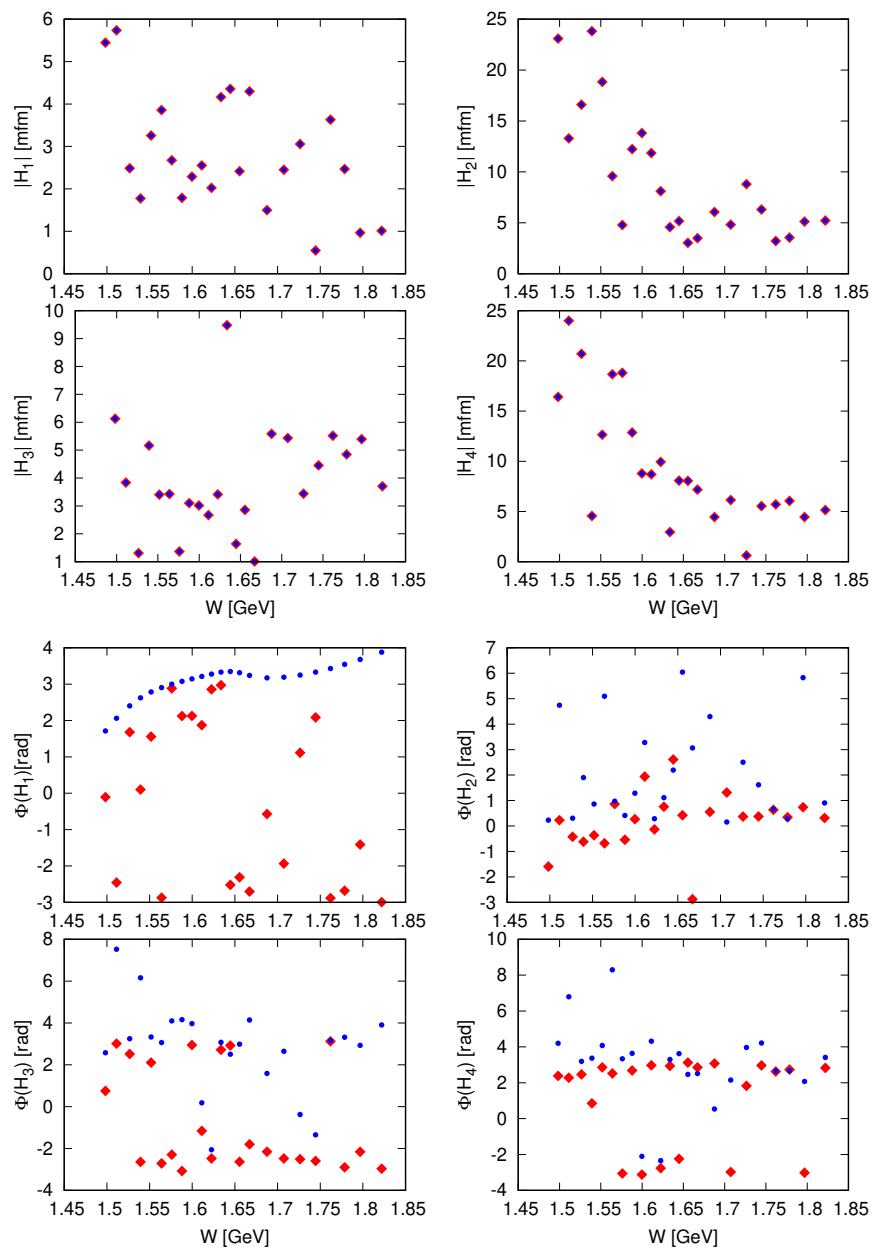

Figure 2: (Color online) Helicity amplitudes at fixed angle for the real data of ref.[2] unconstrained, before the rotation (red symbols) and after the phase rotation with $H_{1}$ defined by Eq. (1) (blue symbols).

We see that the only smooth function is the phase of helicity amplitude $H_{1}$ after the rotation. Absolute values are identical before and after the rotation as they should be, but they are discontinuous as are the phases of helicity amplitudes $\mathrm{H}_{2}-\mathrm{H}_{4}$. The reasons for that are numerous: the used data base is certainly incomplete, but errors in some observables are also rather big, and some data also 
might be mutually incompatible (not proven yet). So, the method used for pseudo-data for real data does not work.

\section{Fixed-t analyticity}

Fixed-t analyticity, the method developed by G. Hoehler in Karlsruhe [20], developed for $\eta$ photoproduction in ref. [2], and with great success applied to $\pi$-nucleon scattering in ref. [3], can easily be applied for imposing the continuity of phase. It is based on the idea that imposing analyticity in $t$ automatically imposes continuity in both, energy and angle as $t$ is in general a function of energy and angle. This is illustrated in Fig. 3 where Mandelstam $(\mathrm{W}, \mathrm{t})$ graph is shown for $\eta$-photoproduction. $W$ is center of mass energy and $t$ is standard Mandelstam variable for $\eta$ photoproduction defined in ref. [2].

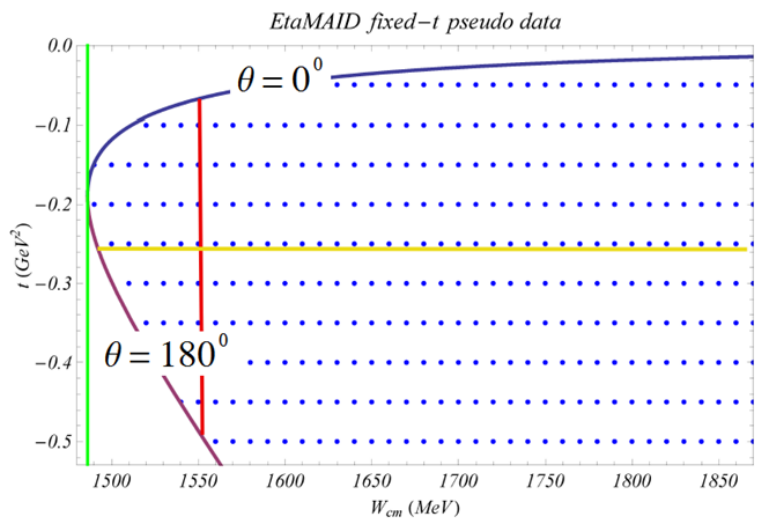

Figure 3: (Color online) Mandelstam $(\mathrm{W}, \mathrm{t})$ graph is shown for $\eta$-photoproduction.

Fixed-W PWA is performed by using the data along the red vertical line, and there is no connection between phases at nearby energies, and as it was demonstrated it causes the discontinuity in energy variation. However, fixed-t PWA is performed along the yellow horizontal line, and analyticity in $t$ automatically imposes continuity on both, angle and energy. So nearby energies are treated simultaneously, and continuity in energy is automatically achieved. Therefore, a two step procedure was invented $[2,20]$ :

\section{Step 1:}

Obtaining a fit of an analytic function to all experimental data at fixed-t which automatically creates continuity in energy and angle.

However, after Step 1 we are still model dependent and are still at the level of reaction amplitudes and DO NOT have partial waves yet! So, we have to introduce Step 2.

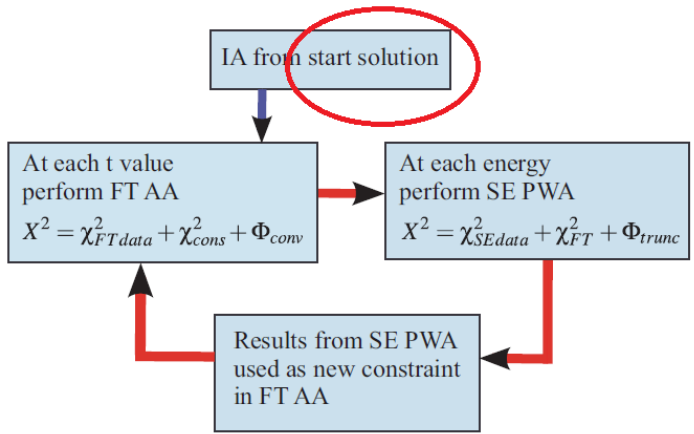

Figure 4: Iterative scheme for the fixed-t minimization. Circle designates the only step of the process which depends on some theoretical assumptions as ETA-MAID15a theoretical parameters are taken as start values for the first minimization. Figure is taken over from ref. [2].

\section{Step 2:}

Create partial waves through a constrained SE PWA using continuity in energy and angle obtained in Step 1 as a penalty function.

In more details, we have to perform the following actions:

Step 1:

- We create the data base in the form $O_{i}=f\left(W_{\text {fixed }}, t\right)$ from the existing experimental data base $O_{i}=f\left(W_{\text {fixed }}, \Theta\right)$.

- Instead of modeling reaction amplitudes as function of $\mathrm{W}$ and $t$ we expand them using Pietarinen variable (Pietarinen expansion [16]).

- We fit the artificial data base $O_{i}=f\left(W_{\text {fixed }}, t\right)$ with these expansions (each reaction amplitude) by fitting coefficients of the Pietarinen expansion, and starting with coefficients of the Pietarinen expansion of the initial solution.

- In this way and obtain a result which automatically analytic in energy and angle, and optimally describes the input. 
Step 2:

We want to obtain continuous SE PWA. This means we have to ensure that neighboring points in energy belong to the same solution (solution having the same phase)! We do it via PENALTY FUNCTION TECHNIQUE.

$$
X^{2}=\chi_{\text {SEdata }}^{2}+\chi_{\mathrm{FT}}^{2}
$$

where $\chi_{\mathrm{FT}}^{2}$ is a constraining term given by

$$
\begin{aligned}
\chi_{\mathrm{FT}}^{2}= & q_{\text {cons }} \sum_{k=1}^{4} \sum_{i=1}^{N^{c}}\left[\frac{\operatorname{Re} H_{k}\left(E, \theta_{i}\right)^{\mathrm{fit}}-\operatorname{Re} H_{k}\left(E, \theta_{i}\right)^{\mathrm{FT}}}{\varepsilon_{k, i}^{\mathrm{Re}}}\right]^{2} \\
& +q_{\text {cons }} \sum_{k=1}^{4} \sum_{i=1}^{N^{c}}\left[\frac{\operatorname{Im} H_{k}\left(E, \theta_{i}\right)^{\mathrm{fit}}-\operatorname{Im} H_{k}\left(E, \theta_{i}\right)^{\mathrm{FT}}}{\varepsilon_{k, i}^{\operatorname{Im}}}\right]^{2}
\end{aligned}
$$

where $F T$ subscript indicates that that the corresponding amplitude is a solution of a fixed-t Pietarinen fit, and $q_{\text {cons }}$ is a tunable penalty-factor.

The whole process is implemented using the iteration scheme depicted in Fig. 4.

So, the process effectively goes like this:

First we perform the minimization in t. To do so, we create the artificial data base at fixed-t. We do it by SPLINE3 fit of existing experimental data to obtain values of observables at the values of angle which is not actually measured, but which is needed for the chosen and fixed value of $t$ at various energies. Then we identify the fitted value with the value of that observable at chosen $t$. This, somewhat artificial data base, is then fitted with a specific form of reaction amplitudes. In our method we use invariant amplitudes, which are calculated from standard helicity amplitudes. To impose analyticity in $t$, we use the conformal mapping generated Pietarinen expansion [2]. Namely, to claim that the real and imaginary part of a function belong to the same analytic function, one has to know the value of the function on the complete real axes in order to be able to calculate the dispersion integral. However, this is never the case for the measured data. So, we are using the following trick: when we fit the data with a Pietarinen expansion of reaction amplitudes, then real and imaginary part by construction satisfy dispersion relations. So, when our fitting parameters are taken to be Pietarinen coefficients, the analyticity is automatically imposed. However, determining initial values of Pietarinen coefficients for reaction amplitudes is non-trivial, and this is the only model-dependent step of our procedure. We take reaction amplitudes from a certain theoretical model (MAID-[21], Bonn-Gatchina[22],Juelich-Bonn-[23],...), and expand them into Pietarinen series. The obtained coefficients are the starting values of our minimization. We perform the minimization and obtain a set of reaction amplitudes which at the same time fit the data, and are analytic in $t$, hence continuous in $\mathrm{W}$ and $\Theta$. The next step is fixed-W PWA constrained with the analytic and continuous reaction amplitudes in $t$, coming from the Step 1. Constraining is defined by set of equations (2) via penalty function technique. The idea is to perform a fit to the data, and obtain a good representation of the data, but at the same time require that the helicity amplitudes be as close to the analytic amplitudes obtained in Step 1 as possible. In that way we get a reasonable representation of data, and approximate analyticity. However, we do not expect that neither the ideal agreement with the data, nor exact analyticity are achieved immediately. Therefore, we introduce iterations. We return the obtained set of helicity amplitudes into Step 1 as initial amplitudes, we fit these amplitudes with Pietarinen expansion, and repeat the Step 1, but this time with amplitudes which are closer to the fitted data set, than energy dependent (ED) model amplitudes of first step are. Then we perform the fit, and go into Step 2 with the obtained result. We expect that in the second step, the obtained result will be closer to the data, and satisfy the analyticity properties better than in first step. And we repeat the procedure as long as we do not get a stable result. At the end of our procedure we obtain the amplitudes (or partial waves) which are as close to the data as possible, and and the same time almost analytic with the analyticity imposed in Step 1 . However, let us observe that there is still is some model dependence is Step 1 as we have prescribed a definite, very simple, form of analyticity by introducing Pietarinen expansion. And with this procedure we create a final set of partial waves. We give the final set of partial waves obtained with this procedure for $\eta$-photoproduction in Fig. 5 (see ref. [2]).

In this result we see four set of partial waves obtained by using two theoretical models to generate initial values: MAID-[21] and Bonn-Gatchina-[22]. Observe that we have two groups of solutions which are obviously different. The reason is that the overall phase of partial wave decomposition for the used models is notably different, and it influences partial waves. For details see ref. [1]. The main message of that paper is that if we want to compare partial waves, we have to match the overall phase, and that has to be done correctly, on the level of amplitudes and not on the level of partial waves. Therefore, we have "rotated" the solution corresponding to the Bonn-Gatchina initial phase to the MAID phase, and solution corresponding to the MAID initial phase to Bonn-Gatchina phase. So, we have four solutions: 1. MAID as initial model with the MAID phase (black squares); 2) MAID as initial model rotated to Bonn-Gatchina phase (green inverted triangles); 3) Bonn-Gatchina as initial model at the BonnGatchina phase (blue triangles); and 4) Bonn-Gatchina as initial model rotated to MAID phase (red circles). We see that in most of partial waves we

see a good agreement between MAID and BonnGatchina results at the same phase, with the exception of one: there is a notable discrepancy in M1- multipole. So, we need new measurements to resolve the uncertainty. This, as seen in Fig. 6 at the randomly chosen and representative energy of $\mathrm{W}=1600 \mathrm{MeV}$, shows that presently used data set is incomplete, and that we need more measurements to clarify the difference. We see that in mea- 

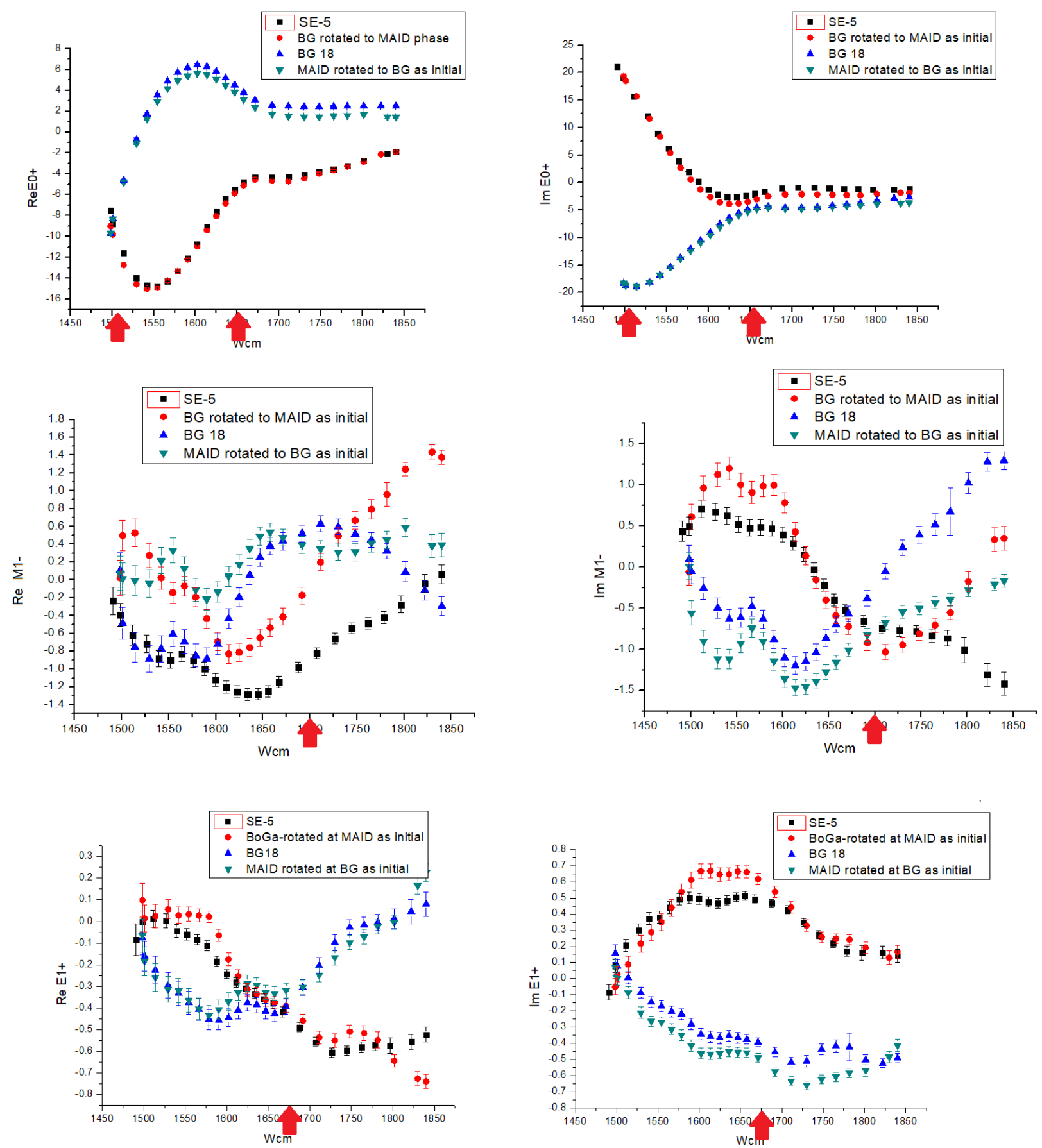

Figure 5: (Color online) Final set of $\eta$-photoproduction partial wave amplitudes obtained in ref. [2] for the lowest multipoles.

sured observables $\mathrm{d} \sigma / \mathrm{d} \Omega, \mathrm{F}, \mathrm{T}$ and $\Sigma$ all four SE solutions agree, while the difference in M1- multipole is notably seen in observables $P, G, H$ and $O_{z}$. Therefore, new measurements are needed.

The situation with overall phase ambiguity is very different for $\pi^{0}$ photoproduction. In $\eta$ photoproduction the overall phase is free, and fixed-t analyticity fixes the phase of SE solution to always different phases determined by the phase of initial solution. So, all four generated SE solutions look notably different. For pion photopro- duction, the phase of all four initial solutions, which is determining the phase of the final SE solution, is fixed by the Watson's theorem [24], and the whole discrepancy can be solely attributed to the data uncertainty. Let us briefly elaborate on that. Since the electromagnetic interaction is in pion photoproduction treated to the first order in the coupling constant, the complexity of the structure functions is completely due to the pion-nucleon interaction. Therefore, each partial wave component $F_{i}^{\alpha}$ of the structure functions may be written in the form 

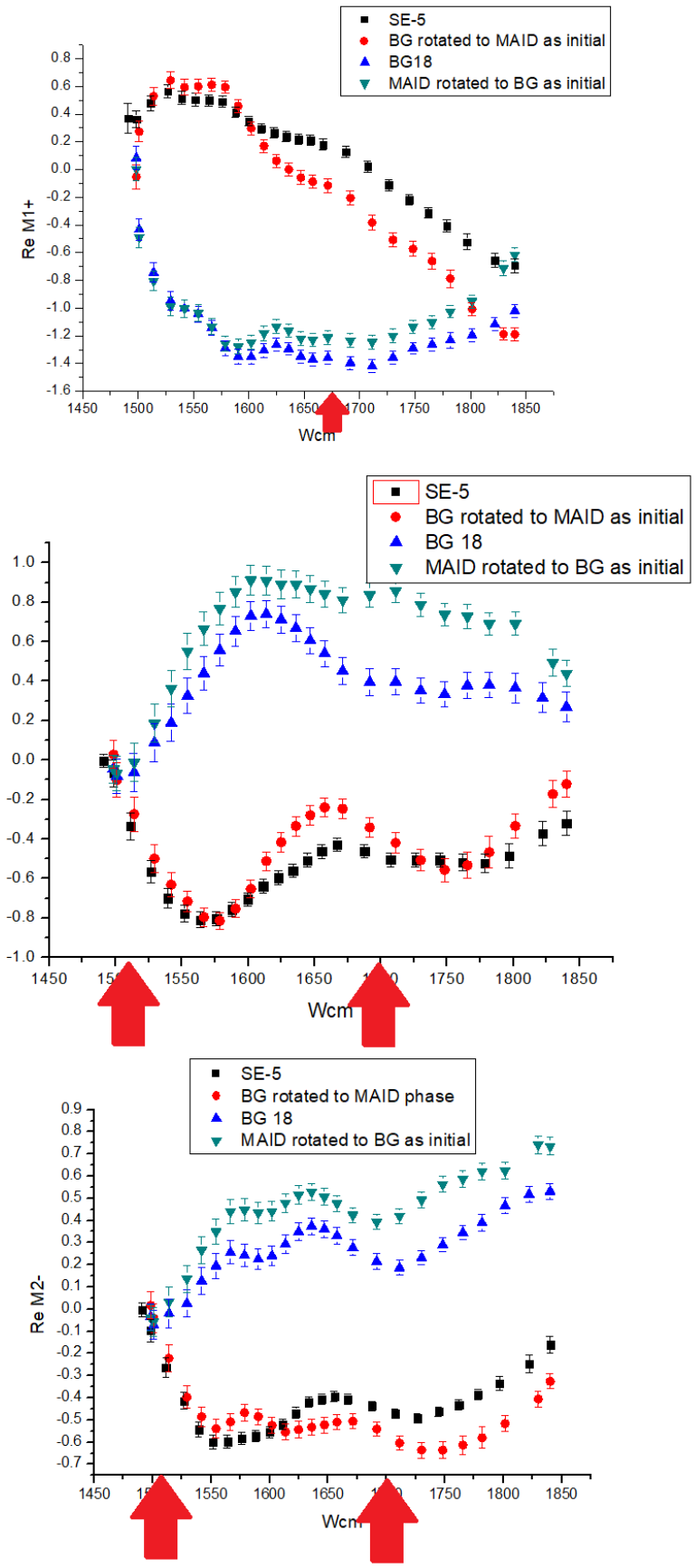
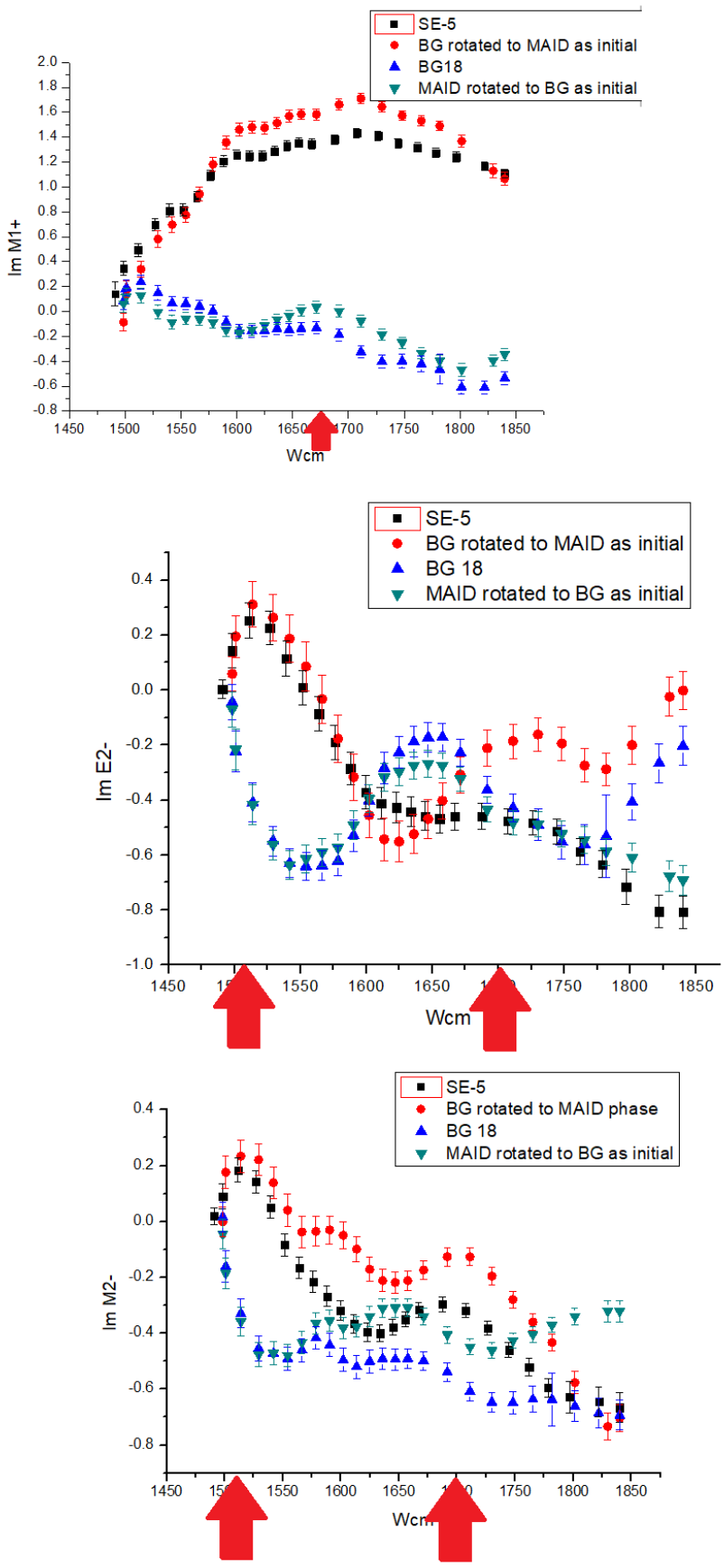

Figure 6: (Color online) Final set of $\eta$-photoproduction partial wave amplitudes obtained in ref. [2] for higher multipoles.

$F_{i}^{\alpha}=e^{\mathrm{i} \delta_{\alpha}} R_{i}^{\alpha}$, where $R_{i}^{\alpha}$ is a real function of the kinematical values and $\delta_{\alpha}$ is the phase shift for elastic $\pi \mathrm{N}$ scattering in that particular channel $\alpha$. Therefore, the phase of pion photoproduction is at lower energies determined by the phase of pion elastic scattering, and that is the condition which is obeyed by all ED solutions. Hence, this unique phase is in all four cases transferred to all four presented SE solutions, and they all must have very similar phase. Therefore, the strong similarity of not only dominant, but of all partial waves in pion photoproduction at lower energies strongly indicate that the data base is here almost complete, but only almost. Some differences among all four solutions, however, indicate that some improvements, as we shall demonstrate later, are still possible.

Therefore, in $\pi^{0}$ photoproduction there is no ambiguity in the overall phase at energies lower than 1650 MeV. In Fig. 7 we show a set of fixed-t solutions for pion photoproduction when MAID-[21], Bonn-Gatchina[22], Jülich-Bonn-[23] and SAID-[25] are taken as initial solutions in the first step of iterative procedure. 


\section{$E=894 \mathrm{MeV}, W=1600 \mathrm{MeV}$}
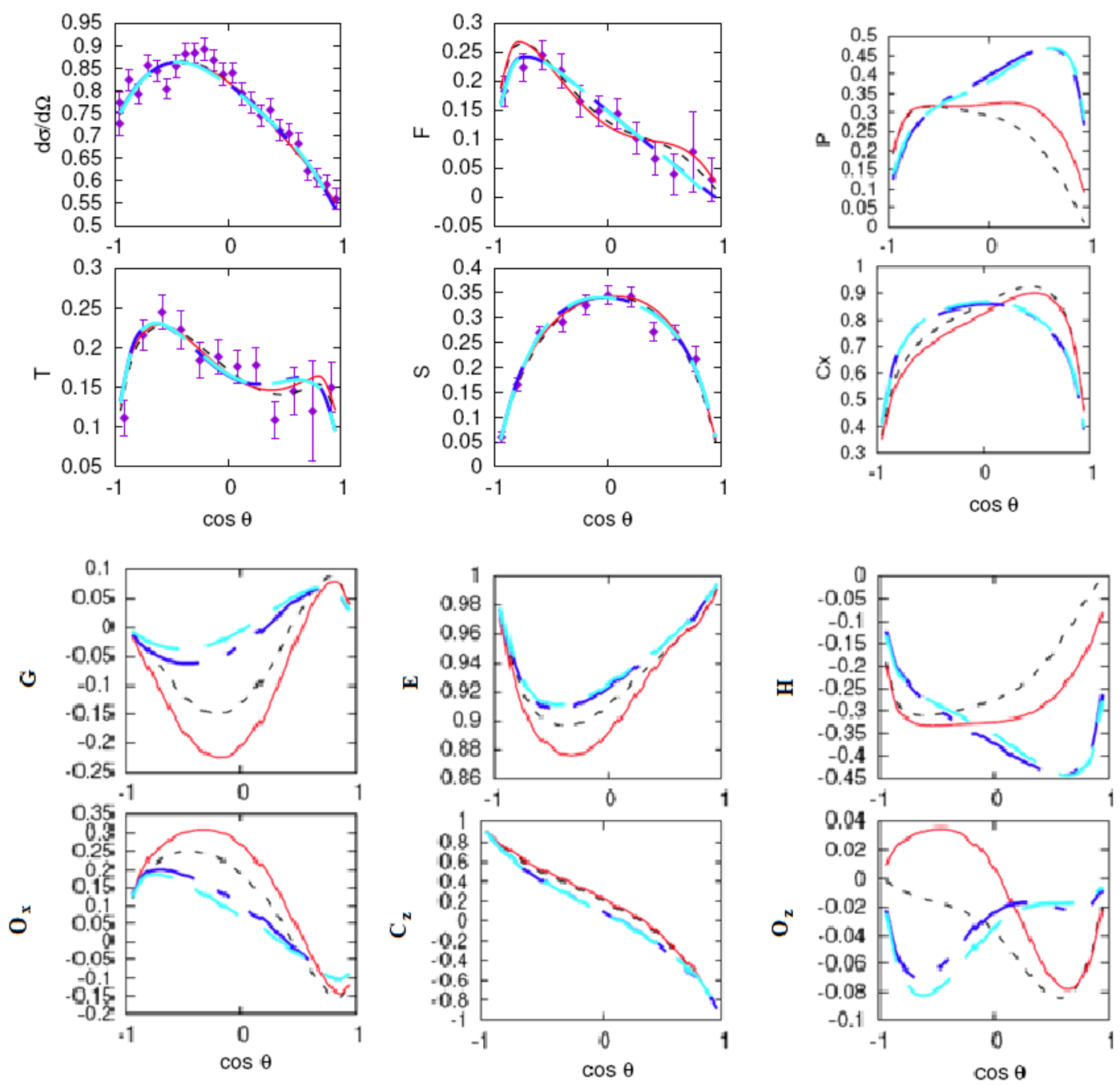

Figure 7: (Color online) The comparison of four obtained SE solutions with measured and unmeasured observables. The colors of the curves shown here correspond to the colors of the single-energy multipoles shown in Figs 5 and 6.

\section{Laurent + Pietarinen expansion}

The driving concept behind the Laurent-Pietarinen $(\mathrm{L}+\mathrm{P})$ expansion was the aim to replace an elaborate theoretical model by a local power-series representation of partial wave amplitudes [16]. The complexity of a partial-wave analysis model is thus replaced by much simpler modelindependent expansion which just exploits analyticity. The $\mathrm{L}+\mathrm{P}$ approach separates pole and regular part in the form of a Laurent expansion, and instead of modeling the regular part in some physical model it uses the conformal mapping to expand it into a rapidly converging power series with well defined analytic properties. So, the method replaces the regular part calculated in a model by the simplest analytic function which has correct analytic proper- ties of the analyzed partial wave (multipole), and fits the data. In such an approach the model dependence is minimized, and is reduced to the choice of the number and location of branch-points used in the $\mathrm{L}+\mathrm{P}$ expansion.

The $\mathrm{L}+\mathrm{P}$ expansion is based on the Pietarinen expansion used in some former papers in the analysis of pion-nucleon scattering data [27-30], but for the $\mathrm{L}+\mathrm{P}$ model the Pietarinen expansion is applied in a different manner. It exploits the Mittag-Leffler expansion ${ }^{1}$ of partial wave amplitudes near the real energy axis, representing the regular, but unknown, background term by a conformal-mapping-generated, rapidly converging power

\footnotetext{
${ }^{1}$ Mittag-Leffler expansion [31]. This expansion is the generalization of a Laurent expansion to a more-than-one pole situation. For simplicity, we will simply refer to this as a Laurent expansion.
} 
Table 1: Formulae defining the Laurent+Pietarinen $(\mathrm{L}+\mathrm{P})$ expansion.

$$
\begin{aligned}
& T^{a}(W)=\quad \sum_{j=1}^{N_{p o l e}} \frac{x_{j}^{a}+\imath y_{j}^{a}}{W_{j}-W}+\sum_{k=0}^{K^{a}} c_{k}^{a} X^{a}(W)^{k}+\sum_{l=0}^{L^{a}} d_{l}^{a} Y^{a}(W)^{l}+\sum_{m=0}^{M^{a}} e_{m}^{a} Z^{a}(W)^{m} \\
& X^{a}(W)=\quad \frac{\alpha^{a}-\sqrt{x_{P}^{a}-W}}{\alpha^{a}+\sqrt{x_{P}^{a}-W}} ; \quad Y^{a}(W)=\frac{\beta^{a}-\sqrt{x_{Q}^{a}-W}}{\beta^{a}+\sqrt{x_{Q}^{a}-W}} ; \quad Z^{a}(W)=\frac{\gamma^{a}-\sqrt{x_{R}^{a}-W}}{\gamma^{a}+\sqrt{x_{R}^{a}-W}} \\
& D_{d p}^{a}=\quad \frac{1}{2 N_{W}^{a}-N_{p a r}^{a}} \sum_{i=1}^{N_{W}^{a}}\left\{\left[\frac{\operatorname{Re} T^{a}\left(W^{(i)}\right)-\operatorname{Re} T^{a, \exp }\left(W^{(i)}\right)}{E r r_{i, a}^{\operatorname{Re}}}\right]^{2}+\left[\frac{\operatorname{Im} T^{a}\left(W^{(i)}\right)-\operatorname{Im} T^{a, \exp }\left(W^{(i)}\right)}{E r r_{i, a}^{\operatorname{Im}}}\right]^{2}\right\}+\mathcal{P}^{a} \\
& \mathcal{P}^{a}=\quad \lambda_{c}^{a} \sum_{k=1}^{K^{a}}\left(c_{k}^{a}\right)^{2} k^{3}+\lambda_{d}^{a} \sum_{l=1}^{L^{a}}\left(d_{l}^{a}\right)^{2} l^{3}+\lambda_{e}^{a} \sum_{m=1}^{M^{a}}\left(e_{m}^{a}\right)^{2} m^{3} \quad D_{d p}=\sum_{a}^{a l l} D_{d p}^{a} \\
& \text { a ..... channel index } \quad N_{\text {pole }} \ldots . . . \text { number of poles } \quad W_{j}, W \in \mathbb{C} \\
& x_{i}^{a}, y_{i}^{a}, c_{k}^{a}, d_{l}^{a}, e_{m}^{a}, \alpha^{a}, \beta^{a}, \gamma^{a} \ldots \in \mathbb{R} \\
& D_{d p}^{a} \ldots . . . \text { discrepancy function in channel } a \\
& N_{W}^{a} \ldots . . . \text { number of energies in channel } a \\
& N_{\text {par }}^{a} \ldots . . . \text { number of fitting parameters in channel } a \\
& \mathcal{P}^{a} \text {..... Pietarinen penalty function } \\
& \lambda_{c}^{a}, \lambda_{d}^{a}, \lambda_{e}^{a} \ldots . . \text { Pietarinen weighting factors } \\
& x_{P}^{a}, x_{Q}^{a}, x_{R}^{a} \in \mathbb{R} \quad(\text { or } \in \mathbb{C}) .
\end{aligned}
$$
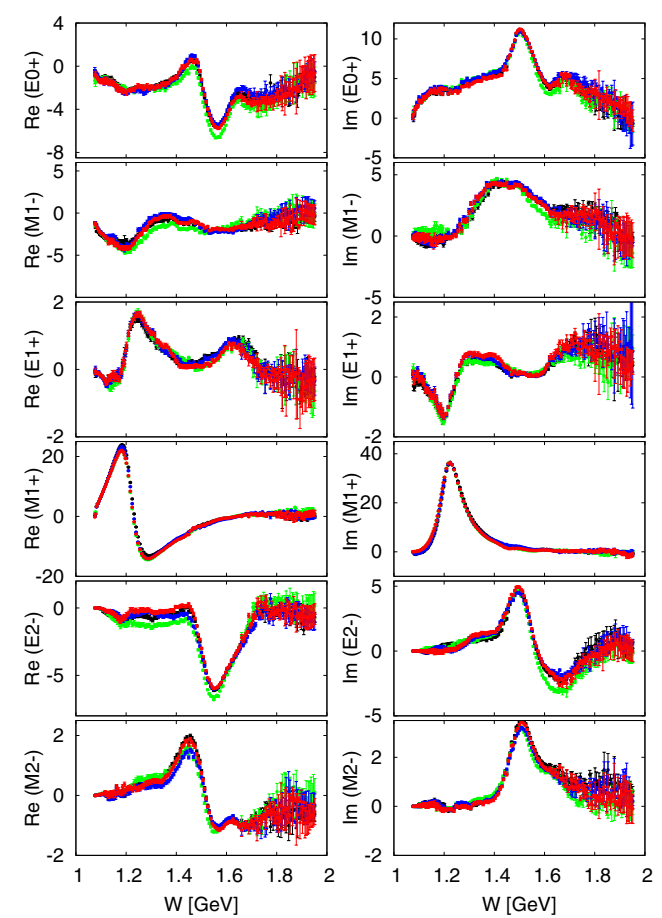

Figure 8: (Color online) SE solutions for the few lowest partial waves obtained using different models as initial solutions (BnGa (black),JuBo (blue), SAID (red) and MAID (green)). Multipoles are in units of $\mathrm{mfm}$. Figure is taken over from ref. [3].

series called a Pietarinen expansion ${ }^{2}$. The method was

\footnotetext{
${ }^{2} \mathrm{~A}$ conformal mapping expansion of this particular type was introduced by Ciulli and Fisher [27, 28], was described in detail and used in pion-nucleon scattering by Esco Pietarinen $[29,30]$. The procedure was denoted as a Pietarinen expansion by G. Höhler in [20].
}

used successfully in several few-body reactions [17, 18, 32], and recently generalized to the multi-channel case [33]. The formulae used in the $\mathrm{L}+\mathrm{P}$ approach are collected in Table 1.

In the fits, the regular background part is represented by three Pietarinen expansion series, all free parameters are fitted. The first Pietarinen expansion with branch-point $x_{P}$ is restricted to an unphysical energy range and represents all left-hand cut contributions. The next two Pietarinen expansions describe the background in the physical range with branch-points $x_{Q}$ and $x_{R}$ respecting the analytic properties of the analyzed partial wave. The second branch-point is mostly fixed to the elastic channel branchpoint, the third one is either fixed to the dominant channel threshold, or left free. Thus, only rather general physical assumptions about the analytic properties are made like the number of poles and the number and the position of branch-points, and the simplest analytic function with a set of poles and branch-points is constructed. The method is applicable to both, theoretical and experimental input, and represents the first reliable procedure to extract pole positions from experimental data, with minimal model bias.

The generalization of the $\mathrm{L}+\mathrm{P}$ method to a multichannel $\mathrm{L}+\mathrm{P}$ method is performed in the following way: i) separate Laurent expansions are made for each channel; ii) pole positions are fixed for all channels, iii) residua and Pietarinen coefficients are varied freely; iv) branch-points are chosen as for the single-channel model; v) the singlechannel discrepancy function $D_{d p}^{a}$ (see Eq. (5) in ref. [32]) which quantifies the deviation of the fitted function from the input is generalized to a multi-channel quantity $D_{d p}$ by summing up all single-channel contributions, and vi) the minimization is performed for all channels in order to obtain the final solution. 


\section{Conclusions}

Unconstrained SE PWA is discontinuous because the continuity of continuum ambiguity phase at neighbouring energies is not ensured. One of the ways to achieve the continuity in energy is imposing fixed-t analyticity. We present four sets of solutions for $\eta$-photoproduction, and four sets of solutions for $\pi^{0}$-photoproduction are presented. Importance of Watson theorem for fixing the phase is stressed for $\pi^{0}$-photoproduction. New methods of imposing the continuity in phase with the final result of getting a continuous PW solution are under development.

\section{Acknowledgment}

This work was supported by the Deutsche Forschungsgemeinschaft (SFB 1044).

\section{References}

[1] A. Švarc, Y. Wunderlich, H. Osmanović, M. Hadžimehmedović, R. Omerović, J. Stahov, V. Kashevarov, K. Nikonov, M. Ostrick, L. Tiator, and R. Workman, Few-Body Syst (2018) 59:96.

[2] H. Osmanović, M. Hadžimehmedović, R. Omerović, J. Stahov, V. Kashevarov, K. Nikonov, M. Ostrick, L. Tiator, and A. Švarc, Phys. Rev. C 97, 015207 (2018).

[3] A. Švarc, H. Osmanović, M. Hadžimehmedović, R. Omerović, J. Stahov, M. Gorchteyn, and V. Kashevarov, K. Nikonov, M. Ostrick, L. Tiator, nucl-tharXiv:1908.05167, Phys. Rev. C 100, 055203 (2019).

[4] A.D. Martin and T.D. Spearman: Elementary Particle Theory, North-Holland Publishing Company, Amsterdam 1970.

[5] https://www.jlab.org/physics

[6] https://www.blogs.uni-mainz.de/fb08-nuclearphysics/accelerators-mami-mesa/the-mainz-microtron/

[7] https://www-elsa.physik.uni-bonn.de/index.html

[8] D. Atkinson, P.W. Johnson and R.L. Warnock, Commun. mat. Phys. 33 (1973) 221.

[9] J.E. Bowcock and H. Burkhard, Rep. Prog. Phys. 38 (1975) 1099.

[10] D. Atkinson and I.S. Stefanescu, Commun. Math. Phys. 101, 291 (1985).

[11] A.V. Anisovich, R. Beck, E. Klempt, V.A. Nikonov, A.V. Sarantsev, U. Thoma, Eur. Phys. J. A48, 15 (2012).

[12] L. Tiator, D. Drechsel, S. S. Kamalov and M. Vanderhaeghen, Eur. Phys. J. ST 198, 141 (2011).

[13] A. M. Sandorfi, S. Hoblit, H. Kamano, and T.-S. H. Lee, J. Phys. G: Nucl. Part. Phys. 38 (2011) 053001.
[14] W. T. Chiang and F. Tabakin, Phys. Rev. C 55, 2054 (1997).

[15] A. Švarc, Y. Wunderlich, H. Osmanović, M. Hadžimehmedović, R. Omerović, J. Stahov, V. Kashevarov, K. Nikonov, M. Ostrich, L. Tiator, R. Workman, Phys. Rev. C 97, 054611 (2018).

[16] A. Švarc, M. Hadzimehmedovic, H. Osmanovic, J. Stahov, L. Tiator, and R. L. Workman, Phys, Rev. C88, 035206 (2013).

[17] A. Švarc, M. Hadzimehmedovic, R. Omerovic, H. Osmanovic, and J. Stahov, Phys, Rev. C89, 0452205 (2014).

[18] A. Švarc, M. Hadzimehmedovic, H. Osmanovic, J. Stahov, L. Tiator, and R. L. Workman, Phys, Rev. C89, 65208 (2014).

[19] V. L. Kashevarov, L. Tiator, M. Ostrick, Bled Workshops Phys., 16, 9 (2015).

[20] G. Höhler, Pion Nucleon Scattering, Part 2, LandoltBornstein: Elastic and Charge Exchange Scattering of Elementary Particles, Vol. 9b (Springer-Verlag, Berlin, 1983).

[21] L. Tiator, M. Gorchteyn, V. L. Kashevarov, K. Nikonov, M. Ostrick, M. Hadžimehmedović, R. Omerović, H. Osmanović, J. Stahov, and A. Švarc v, arXiv:1807.04525v1 [nucl-th], and references therein.

[22] A. V. Anisovich et al., Phys. Rev. C 96, 055202 (2017), and references therein.

[23] D. Rönchen, M. Döring, and U.-G. Meißner, arXiv:1801.10458v1 [nucl-th], and references therein.

[24] K. M. Watson, Phys. Rev. 95, 228 (1954).

[25] For SAID solutions GE09 and E429 see Ref. [26].

[26] E. F. McNicoll et al. [Crystal Ball at MAMI Collaboration], "Study of the $\gamma p->\eta p$ reaction with the Crystal Ball detector at the Mainz Microtron(MAMIC)," Phys. Rev. C 82, 035208 (2010). Erratum: [Phys. Rev. C 84, 029901 (2011)]

[27] S. Ciulli and J. Fischer in Nucl. Phys. 24, 465 (1961).

[28] I. Ciulli, S. Ciulli, and J. Fisher, Nuovo Cimento 23, 1129 (1962).

[29] E. Pietarinen, Nuovo Cimento Soc. Ital. Fis. 12A, 522 (1972).

[30] E. Pietarinen, Nucl. Phys. B107, 21 (1976).

[31] Michiel Hazewinkel: Encyclopaedia of Mathematics, Vol.6, Springer, 31. 8. 1990, pg.251.

[32] A. Švarc, M. Hadžimehmedović, H. Osmanović, J. Stahov, and R. L. Workman, Phys. Rev. C91, 015207 (2015).

[33] A. Švarc, M. Hadžimehmedović, H. Osmanović, J. Stahov, L. Tiator, R. L. Workman, Phys. Lett. B755 (2016) 452-455. 